\title{
Enhanced X-Ray Emission from the Most Radio-powerful Quasar in the Universe's First Billion Years
}

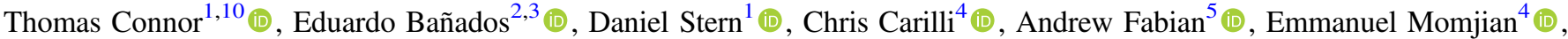 \\ Sofía Rojas-Ruiz ${ }^{2}$ (D), Roberto Decarli ${ }^{6}$ (D), Emanuele Paolo Farina ${ }^{7}$ (i), Chiara Mazzucchelli ${ }^{8}$ (D), and Hannah P. Earnshaw ${ }^{9}$ (iD \\ ${ }^{1}$ Jet Propulsion Laboratory, California Institute of Technology, 4800 Oak Grove Drive, Pasadena, CA 91109, USA; thomas.p.connor@jpl.nasa.gov \\ ${ }^{2}$ Max Planck Institute for Astronomy, Königstuhl 17, D-69117 Heidelberg, Germany \\ ${ }^{3}$ The Observatories of the Carnegie Institution for Science, 813 Santa Barbara Street, Pasadena, CA 91101, USA \\ ${ }^{4}$ National Radio Astronomy Observatory, P.O. Box O, Socorro, NM 87801, USA \\ ${ }^{5}$ Institute of Astronomy, Madingley Road, Cambridge CB3 OHA, UK \\ ${ }^{6}$ INAF-Osservatorio di Astrofisica e Scienza dello Spazio di Bologna, via Gobetti 93/3, I-40129, Bologna, Italy \\ ${ }^{7}$ Max Planck Institut für Astrophysik, Karl-Schwarzschild-Straße 1, D-85748, Garching bei München, Germany \\ ${ }^{8}$ European Southern Observatory, Alonso de Cordova 3107, Vitacura, Region Metropolitana, Chile \\ ${ }^{9}$ Cahill Center for Astronomy and Astrophysics, California Institute of Technology, Pasadena, CA 91125, USA \\ Received 2020 December 9; revised 2021 February 3; accepted 2021 February 15; published 2021 April 23
}

\begin{abstract}
We present deep (265 ks) Chandra X-ray observations of PSO J352.4034-15.3373, a quasar at $z=5.831$ that, with a radio-to-optical flux ratio of $R>1000$, is one of the radio-loudest quasars in the early universe and is the only quasar with observed extended radio jets of kiloparsec scale at $z \gtrsim 6$. Modeling the X-ray spectrum of the quasar with a power law, we find a best fit of $\Gamma=1.99_{-0.28}^{+0.29}$, leading to an X-ray luminosity of $L_{2-10}=1.26_{-0.33}^{+0.45} \times 10^{45} \mathrm{erg} \mathrm{s}^{-1}$ and an X-ray to UV brightness ratio of $\alpha_{\mathrm{OX}}=-1.45 \pm-0.11$. We identify a diffuse structure $50 \mathrm{kpc}\left(\sim 8^{\prime \prime}\right)$ to the $\mathrm{NW}$ of the quasar along the jet axis that corresponds to a $3 \sigma$ enhancement in the angular density of emission and can be ruled out as a background fluctuation with a probability of $P=0.9985$. While with few detected photons the spectral fit of the structure is uncertain, we find that it has a luminosity of $L_{2-10} \sim 10^{44} \mathrm{erg} \mathrm{s}^{-1}$. These observations therefore potentially represent the most distant quasar jet yet seen in X-rays. We find no evidence for excess X-ray emission where the previously reported radio jets are seen (which have an overall linear extent of 0 ". 28), and a bright $\mathrm{X}$-ray point source located along the jet axis to the SE is revealed by optical and NIR imaging to not be associated with the quasar.
\end{abstract}

Unified Astronomy Thesaurus concepts: X-ray quasars (1821); X-ray astronomy (1810); Radio loud quasars (1349); Quasars (1319); Jets (870)

\section{Introduction}

The evolution of supermassive black holes (SMBHs) in the early universe represents a challenge for modern cosmology, requiring significant, sustained growth from primordial seeds to explain the population of observed quasars in the first billion years of the universe (e.g., Inayoshi et al. 2020). In the past decade, not only has the number of known members of this population expanded through large surveys (e.g., Bañados et al. 2016; Jiang et al. 2016; Mazzucchelli et al. 2017; Reed et al. 2017; Matsuoka et al. 2019; Wang et al. 2019; Yang et al. 2019), but individual discoveries have pushed out the extremes of mass (Wu et al. 2015; Yang et al. 2020), luminosity (Pons et al. 2019; Medvedev et al. 2020), and redshift (Mortlock et al. 2011; Bañados et al. 2018c; Wang et al. 2021b) that must be accounted for by theoretical models. Grappling with this challenge requires not only measuring quasar accretion rates, but also identifying mechanisms being used to produce massive growth.

One of the best ways to study this evolution is through X-ray observations, where the emission is produced in the innermost regions of the active galactic nucleus (AGN; Fabian 2016) and where, at high redshifts, the observed energies are less sensitive to intervening obscuration. X-ray observations have been effective at investigating even the most distant known quasars (Bañados et al. 2018b), and the results of X-ray studies of the

\footnotetext{
${ }^{10}$ NPP Fellow.
}

high-redshift population include detection of variability (Nanni et al. 2018) and dual AGN (Connor et al. 2019; Vito et al. 2019a). Recent works by Vito et al. (2019b) and Wang et al. (2021a) have also constrained the evolution of accretion physics for this population of SMBHs; they note a potential steepening of the average X-ray power-law emission at high redshifts $(z \gtrsim 6)$, suggestive of more rapid mass gain at the earliest epochs (e.g., Brightman et al. 2013). Clearly, further study of high-redshift quasars is important for deepening our understanding of early SMBH growth, particularly when these studies expand the parameter space of analyzed quasar properties.

Radio-loud quasars are an important subpopulation of highredshift quasars for understanding early SMBH growth and evolution. Radio-loud refers to quasars with rest-frame $5 \mathrm{GHz}$ flux densities significantly greater than rest-frame optical flux densities; more formally, those quasars with radio-loudness parameter $R=f_{\nu}(5 \mathrm{GHz}) / f_{\nu}(4400 \AA) \gtrsim 10$ (Kellermann et al. 1989). Although the fraction of radio-loud quasars remains consistent with redshift ( $\sim 10 \%$; Bañados et al. 2015), these objects remain effectively unstudied at high redshift in X-ray wavelengths (see Vito et al. 2019b). As such, characterizing the AGN properties of the earliest radio-loud quasars is a crucial step in revealing quasar growth modes.

One quasar of particular interest is PSO J352.4034-15.3373 (hereafter PJ352-15), a radio-loud quasar at $z=5.84 \pm 0.02$ first reported by Bañados et al. (2018a). At its discovery, PJ352 -15 was the radio-loudest quasar known at redshifts $z \gtrsim 6$ by 
Table 1

Chandra Observations

\begin{tabular}{lccc}
\hline \hline Obs ID & $\begin{array}{c}\text { Exposure Time } \\
(\mathrm{ks})\end{array}$ & $\begin{array}{c}\text { Start Date } \\
(\text { YYYY mm dd })\end{array}$ & $\begin{array}{c}\text { Roll Angle } \\
\left({ }^{\circ}\right)\end{array}$ \\
\hline 21415 & 41.52 & 2019 Aug 19 & 88 \\
21416 & 19.06 & 2019 Sep 16 & 359 \\
22728 & 59.28 & 2019 Aug 21 & 88 \\
22729 & 45.46 & 2019 Aug 24 & 88 \\
22730 & 38.24 & 2019 Aug 25 & 88 \\
22850 & 31.44 & 2019 Sep 17 & 359 \\
22851 & 29.88 & 2019 Sep 22 & 350 \\
\hline
\end{tabular}

an order of magnitude, with $R>1000$, although a recently discovered blazar at $z=6.10 \pm 0.03$ has a similar radioloudness (Belladitta et al. 2020). High-resolution very long baseline interferometry (VLBI) radio imaging using the Very Long Baseline Array (VLBA) revealed the presence of linear structure at the quasar's position over $1.62 \mathrm{kpc}(0$ !" 28$)$, divided into three distinct components (Momjian et al. 2018). Whether these structures originate from a radio core with a one-sided jet or instead indicate a compact symmetric object is unclear with the currently published data, ${ }^{11}$ but it is clear that in addition to being radio-loud, PJ352-15 also hosts kiloparsec-extended radio jets. Because of these properties, PJ352-15 is an excellent target for X-ray analysis; indeed, as discussed below, $\mathrm{X}$-rays are potentially the best mechanism for detecting extended jet structures at this redshift (Fabian et al. 2014).

In this work, we present X-ray observations of PJ352-15 with Chandra. We discuss our observations in Section 2 and the X-ray properties of PJ352-15 in Section 3. In Section 4, we introduce the concept of inverse Compton (IC) emission from the cosmic background and detail several methods we used to detect this emission. We further describe the properties of the detected extended emission in Section 5. Finally, we contextualize these results in Section 6. Throughout this work, we adopt a quasar redshift of $z=5.831$ based on observed [C II] $\lambda 158 \mu \mathrm{m}$ emission (S. Rojas-Ruiz et al. 2021, in preparation) and a Galactic neutral hydrogen column density of $N_{\mathrm{H}}=1.68 \times 10^{20} \mathrm{~cm}^{-2}$ in the direction of PJ352-15 (HI4PI Collaboration et al. 2016). We use a flat cosmology with $H_{0}=70 \mathrm{~km} \mathrm{~s}^{-1} \mathrm{Mpc}^{-1}, \Omega_{M}=0.3$, and $\Omega_{\Lambda}=0.7$; the scale at this redshift is $5.80 \mathrm{kpc} \operatorname{arcsec}^{-1}$. All distances given are in proper distances and errors are reported at the $1 \sigma(68 \%)$ confidence level unless otherwise stated.

\section{Observations and Data Reduction}

We observed PJ352-15 with the Advanced CCD Imaging Spectrometer (ACIS; Garmire et al. 2003) on Chandra. Observations were conducted across seven separate visits spread across five weeks, with a total exposure time of $264.88 \mathrm{ks}$. Details of the seven visits are given in Table 1. In all observations, events were recorded in the Very Faint telemetry format and with the Timed Exposure mode. Chandra was positioned so that PJ352-15 appeared on the back-illuminated S3 chip during our observations.

Data reduction was performed using the Chandra Interactive Analysis of Observations software package (CIAO; Fruscione et al. 2006) v4.11 with CALDB version 4.8.4.1. Reduction

\footnotetext{
11 Follow-up multifrequency VLBI analysis should address this ambiguity (E. Momjian et al. 2021, in preparation).
}

followed standard procedures (e.g., Connor et al. 2020), beginning with reprocessing using the chandra_repro script with standard grade, status, and good time filters and with VFAINT background cleaning. As part of the standard reprocessing, events were processed with the Energy Dependent Subpixel Event Repositioning (EDSER) routine ( $\mathrm{Li}$ et al. 2004); because of this reprocessing of the on-axis observations, and to enable more detailed physical modeling, all X-ray images in this work are presented at half-pixel (0."246) resolution (and all images presented are of the combined seven observations). To allow for accurate spatial analysis by minimizing positional uncertainties, we first aligned all observations with Obs ID 22728, our deepest exposure. This alignment was done with a combination of WAVDETECT (Freeman et al. 2002) and the CIAO tools wCS_align and wCS_update.

We used the CIAO script merge_obs to generate coadded images from the seven observations. Images were generated in the soft $(0.5-2.0 \mathrm{keV})$, hard $(2.0-7.0 \mathrm{keV})$, and broad $(0.5-7.0 \mathrm{keV})$ bands; the image of PJ352-15 in the broad band is shown in the left panel of Figure 1. No significant structure is readily apparent within $\sim 15^{\prime \prime}(\sim 85 \mathrm{kpc})$ of the quasar. For spectroscopic analysis of PJ352-15, we use a 2!"0 circular aperture centered on the coordinates from Bañados et al. (2018a), which align with the X-ray centroid (Figure 1). The background was extracted from a concentric annulus with inner and outer radii of $25^{\prime \prime}$ and $38^{\prime \prime}$, respectively. Source and background spectra were created with specextract.

\section{X-Ray Properties of P352-15}

We detect PJ352-15 in a 2 ". 0 radius aperture with $120.3_{-11.2}^{+12.3}$ net counts in the broad band $(0.5-7.0 \mathrm{keV}), 80.9_{-9.1}^{+10.1}$ counts in the soft band $(0.5-2.0 \mathrm{keV})$, and $39.3_{-6.6}^{+7.6}$ counts in the hard band (2.0-7.0 keV), with all uncertainties calculated using the method of Gehrels (1986). From these values, and using the Bayesian methodology described by Park et al. (2006), we derive a hardness ratio, $\mathcal{H} \mathcal{R}^{12}$ for $\mathrm{PJ} 352-15$ of $\mathcal{H} \mathcal{R}=-0.34_{-0.09}^{+0.08}$. As a simple flux ratio, the hardness ratio allows for an easy comparison between sources with few detected counts, as is common for high-redshift quasars (Vito et al. 2019b). However, for comparisons between quasars observed with different observatories or at different times, the relative differences in effective area need to be considered, and a spectral fit, when available, is thus more informative.

Spectroscopic analysis was performed using the Pythonbased implementation of XSPEC v12.10.1 (Arnaud 1996), PyXspec. We did not bin our spectrum, and instead used the modified C-Statistic ( $C$; Cash 1979; Wachter et al. 1979) to find the parameters of the best fit. We fit the spectrum with a multiplicative combination of a power law and Galactic foreground absorption using the XSPEC model phabs $\times$ powerlaw. Here, the Galactic absorption $N_{\mathrm{H}}$ was frozen at its adopted value $\left(1.68 \times 10^{20} \mathrm{~cm}^{-2}\right)$, but the slope and normalization of the power law were allowed to vary. With two free parameters, $1 \sigma$ uncertainties include all values with C-statistic values within $\Delta C \leqslant 2.30$ of the overall best fit. We used the Monte Carlo routines within XSPEC to evaluate the $1 \sigma$ uncertainties of all parameters.

\footnotetext{
$12 \mathcal{H} \mathcal{R}=(H-S) /(H+S)$, where $H$ and $S$ are the net counts in the hard $(2.0-7.0 \mathrm{keV})$ and soft $(0.5-2.0 \mathrm{keV})$ bands, respectively.
} 


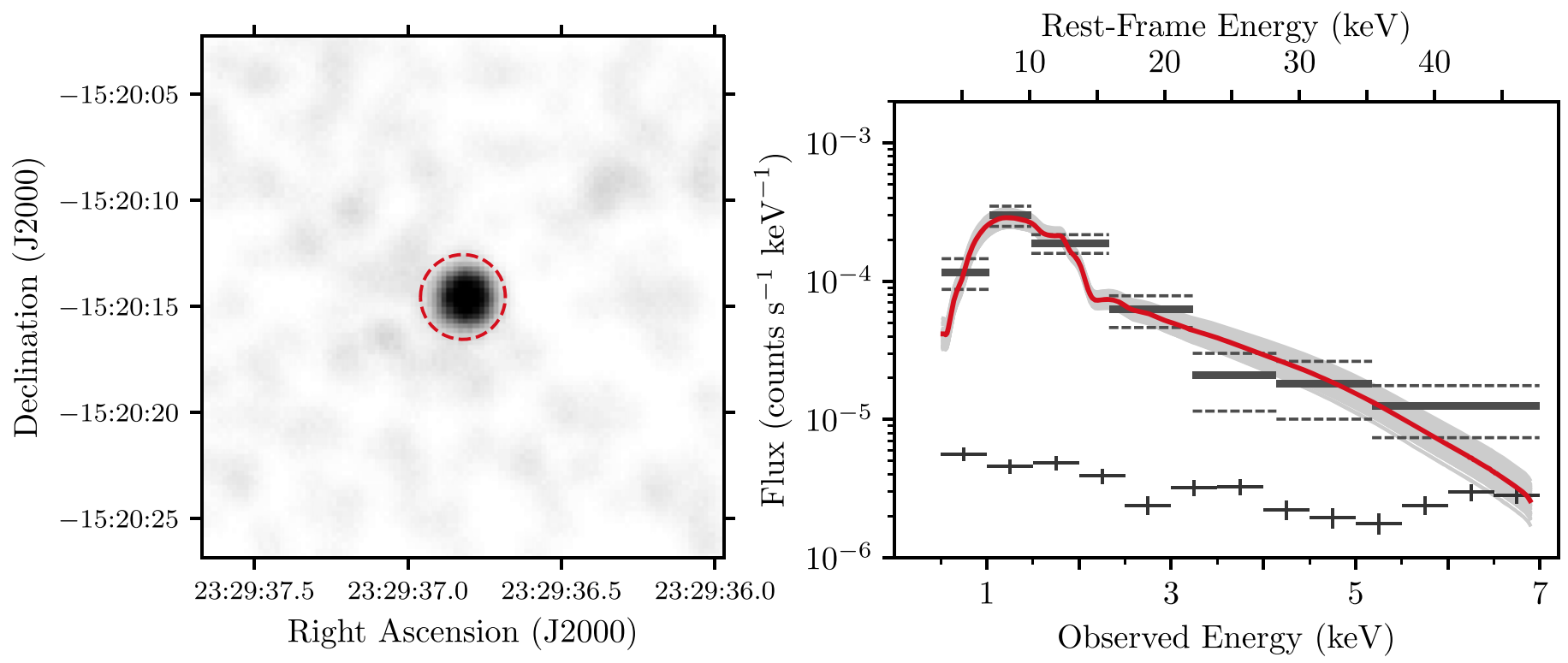

Figure 1. Left: $0.5-7.0 \mathrm{keV}$ Chandra observation of PJ352-15, smoothed by a Gaussian kernel of width 0 ". 75 . The 2 ." 0 radius extraction region used for photometry and spectroscopy is indicated by the red circle. Right: X-ray spectrum of PJ352-15. Data (dark gray) are binned for ease of display, but were not binned during fitting. The best-fitting spectrum is shown in red, while 100 spectra with $\Delta C \leqslant 2.30$ from our Monte Carlo analysis are shown in gray. The background spectrum is shown in dark gray error bars at the bottom.

From our spectral fitting, we find the emission is characterized by a power law of slope $\Gamma=1.99_{-0.28}^{+0.29}$. This value is typical for quasars even up to $z \sim 6$ (Nanni et al. 2018). Including the uncertainties in the normalization of the power law, this translates to a rest-frame $2.0-10.0 \mathrm{keV}$ unabsorbed luminosity of $L_{2-10}=1.26_{-0.33}^{+0.45} \times 10^{45} \mathrm{erg} \mathrm{s}^{-1}$. This best fit is shown in Figure 1, as are 100 of the spectra explored by our Monte Carlo analysis that have $\Delta C \leqslant 2.30$. The $\mathrm{X}$-ray properties of the quasar are summarized in Table 2.

\section{Evidence for Inverse Compton/Cosmic Microwave Background}

PJ352-15 is the source of the most distant extended (kiloparsec-scale) radio jets yet seen (Momjian et al. 2018; but see also Spingola et al. 2020), and it is therefore an ideal candidate to investigate the potential for IC interactions between relativistic particles in jets and the cosmic microwave background (CMB). IC/CMB has long been associated with the X-ray emission seen with jets, dating back to the first observations with Chandra (Chartas et al. 2000; Schwartz et al. 2000), although a number of issues have been raised against possible detections of this effect at low redshifts (Meyer et al. 2015; Breiding et al. 2017).

As a simple model, IC/CMB occurs when a relativistic particle in a jet interacts with a CMB photon; the interaction depletes energy from the jets while also scattering the $\mathrm{CMB}$ photons to X-ray energies. The energy density of the CMB scales as $(1+z)^{4}$, meaning that not only is the effect of cosmological dimming countered, but at higher redshifts it becomes more likely that the CMB should dominate over magnetic fields in lobes as a mechanism for particles to radiate energy. Jets can extract rotational energy from the accretion disk (e.g., Blandford \& Payne 1982), meaning that jets can enable accretion beyond the Eddington limit, and, in the context of high-redshift quasars, allow for more rapid growth (Ghisellini et al. 2013). While the dearth of radio jets at large redshifts has been noted (Ghisellini et al. 2015), Fabian et al. (2014)
Table 2

X-Ray Properties

\begin{tabular}{lcc}
\hline \hline Parameter & Value & Units \\
\hline Net Counts & $120.3_{-11.2}^{+12.3}$ & $\cdots$ \\
Soft Counts & $80.9_{-9.1}^{+10.1}$ & $\cdots$ \\
Hard Counts & $39.3_{-6.6}^{+7.6}$ & $\ldots$ \\
$\mathcal{H} \mathcal{R}$ & $-0.34_{-0.09}^{+0.08}$ & $\ldots$ \\
$\Gamma$ & $1.99_{-0.28}^{+0.29}$ & $\cdots$ \\
$L_{2-10}$ & $1.26_{-0.33}^{+0.45} \times 10^{45}$ & $\mathrm{erg} \mathrm{s}^{-1}$ \\
$F_{0.5-2.0}$ & $2.8_{-0.5}^{+0.6} \times 10^{-15}$ & $\mathrm{erg} \mathrm{s}^{-1} \mathrm{~cm}^{-2}$ \\
$F_{0.5-7.0}$ & $5.5_{-0.8}^{+0.9} \times 10^{-15}$ & $\mathrm{erg} \mathrm{s}^{-1} \mathrm{~cm}^{-2}$ \\
$L_{\nu}(2 \mathrm{keV})$ & $1.62_{-0.66}^{+1.11} \times 10^{27}$ & $\mathrm{erg} \mathrm{s}^{-1} \mathrm{~Hz}^{-1}$ \\
$L_{\nu}(2500 \AA)$ & $9.8_{-1.1}^{+1.2} \times 10^{30}$ & $\mathrm{erg} \mathrm{s}^{-1} \mathrm{~Hz}^{-1}$ \\
$\alpha_{\mathrm{OX}}$ & $-1.45 \pm 0.11$ & $\cdots$ \\
\hline
\end{tabular}

proposed that if jets are primarily emitting through IC/CMB in the early universe, this could allow the growth of observed $z \gtrsim 7$ SMBHs from stellar mass seeds. Direct detection of this effect is therefore of great importance to the understanding of SMBH growth.

In recent years, a number of studies have begun looking for extended X-ray emission around high-redshift AGN, but clear evidence has not yet been seen in the first billion years of the universe. Paliya et al. (2020) reported detecting seven blazars at $3.1 \lesssim z \lesssim 4.7$ with extended emission seen by Chandra, and Napier et al. (2020) detected two extended structures on opposite angles of a $z=4.26$ radio galaxy. While Nanni et al. (2018) identified a potential extended component associated with a $z=6.31$ quasar, further analysis by Gilli et al. (2019) found that the reported emission is associated with a foreground structure. Finally, Fabian et al. (2014) reported two potential structures around a $z=7.1$ quasar, but were unable to rule out this being caused by source confusion in the XMM-Newton observations. In this section, we describe our efforts to search for extended X-ray emission around 
PJ352-15, thereby probing potential jet-assisted growth of early quasars.

\subsection{Presence of Extended Structure}

In addition to looking for potential X-ray signatures of the radio-detected jets, we also looked for possible indications of an X-ray bright jet extending beyond the radio emission, which is roughly the size of a single ACIS pixel. Such X-ray structures have long been seen around some AGN (for a review, see Harris \& Krawczynski 2006). While X-ray components often overlap with radio emission in AGN jets (e.g., Jimenez-Gallardo et al. 2020), this is not always the case; Schwartz et al. (2020), for example, recently reported X-ray detected jet candidates around $z \sim 3.2$ quasars with no corresponding radio emission. Likewise, a serendipitous discovery of a quasar X-ray jet at $z=2.5$ by Simionescu et al. (2016) extends for $\sim 100 \mathrm{kpc}$, while radio emission is only seen out to $\lesssim 10 \mathrm{kpc}$ from the central AGN. In the context of $\mathrm{IC} / \mathrm{CMB}$, for a jet of fixed magnetic field, the relative flux densities between $\mathrm{X}$-ray and radio emission scales as $S_{\mathrm{X}} / S_{\mathrm{r}} \propto(1+z)^{4}$ (Carilli \& Taylor 2002), and so, at higher redshift, the X-ray flux will become easier to detect than the radio component. Conversely, it is also not uncommon for radio and X-ray components of jets to be spatially offset, implying their emission is generated from synchrotron emission from separate populations of jetted particles (e.g., Reddy et al. 2021); however, for such a scenario, we would expect the $\mathrm{X}$-ray component to be stronger upstream in the jet relative to the radio components over large scales (e.g., Siemiginowska et al. 2007).

As a cursory inspection of the area around the quasar in $\mathrm{X}$-rays (shown in Figure 1) does not reveal any structure, we turn to a statistical test to search for excess emission oriented along the jet axes. If present, X-ray jets would manifest as additional X-ray emission outside of the quasar point-spread function (PSF; see Section 4.3) and at position angles of $\sim-60^{\circ}$ and $/$ or $\sim 120^{\circ}$, the angle and counter-angle of the jets reported by Momjian et al. (2018). To quantify this, we calculate $\rho(\theta)$, the azimuthal X-ray density profile (e.g., Connor et al. 2018), summing over all X-ray events such that

$$
\rho(\theta)=\sum_{i} W_{i}(\theta)
$$

Here $W_{i}(\theta)$ is a Boolean value that evaluates to 1 if event $i$ is within an angular wedge centered on angle $\theta$ and within the wedge's inner and outer radii and to 0 otherwise. The wedge is defined by its opening angle $\left(\phi=10^{\circ}\right)$ and inner $\left(r_{i}=5\right.$ !" 0$)$ and outer $\left(r_{o}=12\right.$ ". 0$)$ radii.

Although these observations were processed with EDSER to allow for event positioning to better precision than the size of an ACIS pixel, there is still an inherent uncertainty in where events were recorded. Based on the work of Li et al. (2003), we assume that all events have an inherent uncertainty in position of $\delta=0$ ". 125 in both axes despite their repositioning. As such, we set $W_{i}(\theta)=1$ for events that, if shifted within $\pm \delta$ in $X$ and/ or $Y$ positions, would fall within the wedge. The total area of the wedge is thus

$$
A_{\mathrm{W}}=\frac{\phi \pi}{360^{\circ}}\left(\left(r_{o}+\delta\right)^{2}-\left(r_{i}-\delta\right)^{2}\right)+2 \delta\left(r_{o}+2 \delta-r_{i}\right) .
$$

Therefore, with an expected background of surface brightness $\Omega_{B}$ (in units of counts $\operatorname{arcsec}^{-2}$ ), the background subtracted azimuthal density is

$$
\rho(\theta)-\rho_{B}=\sum_{i} W_{i}(\theta) / A_{\mathrm{W}}-\Omega_{B} .
$$

Uncertainties on this value are estimated by bootstrap resampling of all events that fall within the inner and outer radii of the annulus of interest, including the $\delta$ term. The distribution of the azimuthal X-ray emission density is shown in Figure 2.

A clear peak is seen in Figure 2, corresponding to a position angle of $-55^{\circ} \mathrm{E}$ of $\mathrm{N}$. In comparison, the jets seen by Momjian et al. (2018) are at $-61^{\circ} \mathrm{E}$ of N. From the bootstrapped uncertainties, this excess is a $3 \sigma$ detection of faint structure (top panel of Figure 2). It should be noted that these uncertainties do not include the effects of systematic choices - the values of $r_{i}$, $r_{o}$, and $\phi$, as well as the energy range used. However, we find qualitatively similar $(\gtrsim 3 \sigma)$ results when using an annulus of either 3 !" $0-15$ !. 0 or 5 !" $0-10$ !" 0 or when setting $\phi \in\left[5^{\circ}, 12^{\circ}\right]$. Similarly, relaxing the binary restriction on $W_{i}(\theta)$, by setting $W_{i}(\theta)=0.5$ and $W_{i}(\theta)=1.0$ for photons in the wedge with and without including positional uncertainties, respectively, produces no meaningful change in our results. Due to the paucity of counts, the significance of this detection is maximized in the broad energy band $(0.5-7.0 \mathrm{keV})$, but the peak remains at $2 \sigma-3 \sigma$ significance in the soft and hard bands.

Enhanced X-ray emission is only seen in the NW direction of the quasar; in the context of other X-ray imaging studies, the lack of emission from the counter-jet is common. In a fluxlimited sample of 56 quasars with radio jets at $z \lesssim 2$, Marshall et al. (2018) report 33 have X-ray jets but none have visible counter-jets. While tentative hints of X-ray emission from jets have been seen in quasars up to redshifts $z \lesssim 4.7$ (Paliya et al. 2020; Napier et al. 2020), and from jets of similar sizes $(\gtrsim 50 \mathrm{kpc}$ ) up to redshifts $z \lesssim 3.7$ (Simionescu et al. 2016; Worrall et al. 2020), this observation represents the most distant quasar with $\gtrsim 3 \sigma$ evidence for a projected X-ray jet.

\subsection{X-Ray Loudness}

We also evaluate $\alpha_{\mathrm{OX}}$, the logarithmic ratio of monochromatic luminosities between rest-frame $\mathrm{X}$-ray and $\mathrm{UV}^{13}$ for $\mathrm{PJ} 352-15$. We calculate $L_{\nu}(2 \mathrm{keV})$ directly from the best-fit power law, accounting for redshift corrections following Stern et al. (2000), finding $L_{\nu}(2 \mathrm{keV})=1.62_{-0.66}^{+1.11} \times 10^{27} \mathrm{erg} \mathrm{s}^{-1} \mathrm{~Hz}^{-1}$. To calculate $L_{\nu}(2500 \AA)$, we use the $\mathrm{AB} 1450 \AA$ absolute magnitude given by Bañados et al. (2018a), $M_{1450}=-25.59 \pm 0.13$, and scale that to $2500 \AA$ assuming $f_{\nu} \propto \nu^{\alpha_{\nu}}$, where, per Bañados et al. (2018a), we adopt $\alpha_{\nu}=-0.5$. From this, we calculate $L_{\nu}(2500 \AA)=9.8_{-1.1}^{1.2} \times 10^{30} \mathrm{erg} \mathrm{s}^{-1} \mathrm{~Hz}^{-1}$ and a corresponding value of $\alpha_{\mathrm{OX}}=-1.45 \pm 0.11$.

A number of observational studies have shown a relationship between $L_{\nu}(2500 \AA)$ and $\alpha_{\mathrm{OX}}$, such that, for increasing ultraviolet monochromatic luminosity, the relative strength of the X-ray luminosity declines (e.g., Vignali et al. 2003; Strateva et al. 2005; Steffen et al. 2006). Using the best-fit scaling relation for radio-quiet quasars of Lusso \& Risaliti (2016), we would expect a value of $\alpha_{\mathrm{OX}}=-1.65$ given the ultraviolet luminosity of PJ352-15. Our measured value is

\footnotetext{
${ }^{13} \alpha_{\mathrm{OX}}=0.3838 \times \log \left(L_{\nu}(2 \mathrm{keV}) / L_{\nu}(2500 \AA)\right)$, where $L_{\nu}$ is the monochromatic luminosity.
} 

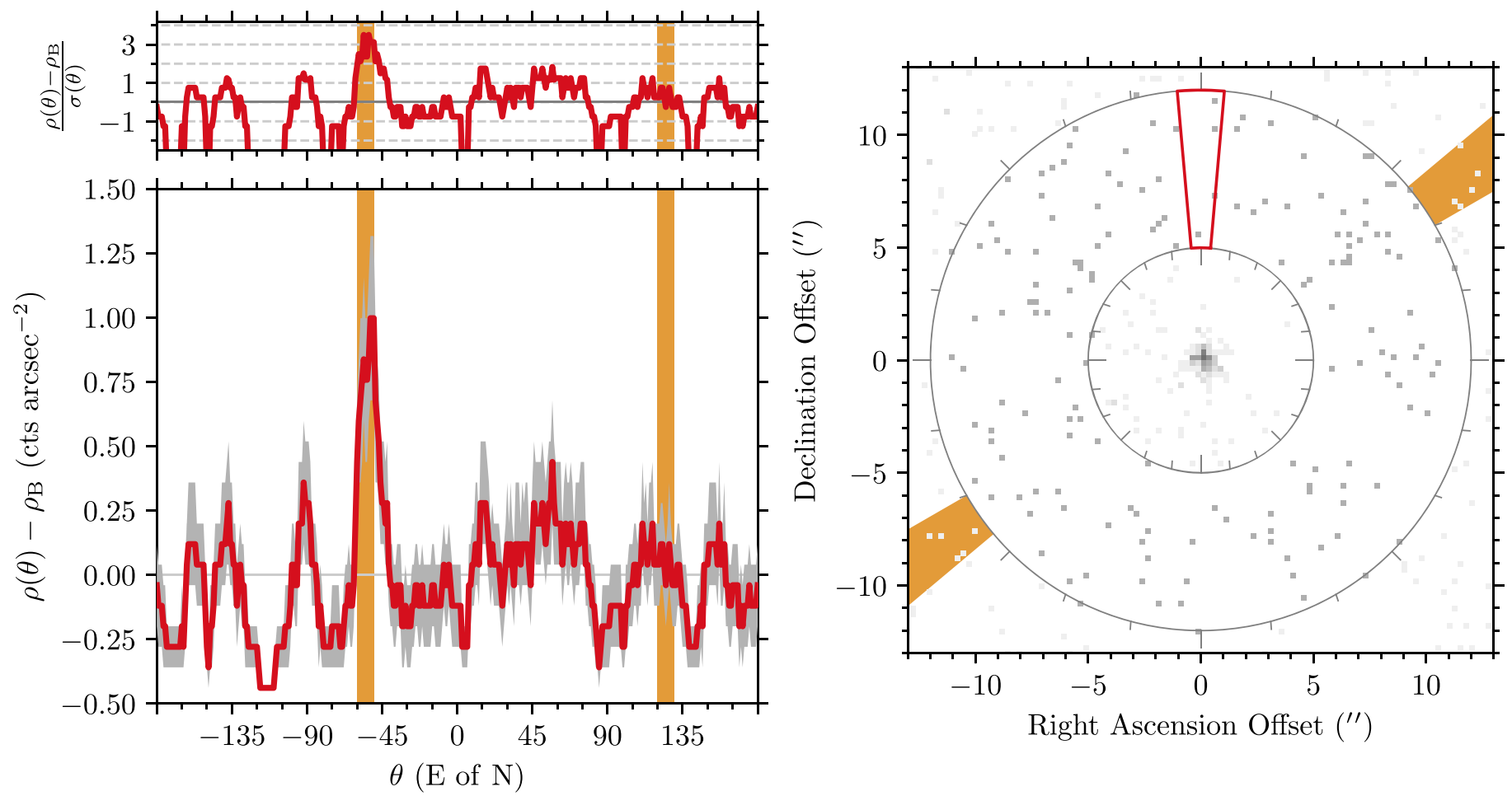

Figure 2. Left: angular overdensity of broad $(0.5-7.0 \mathrm{keV})$ emission in an annulus of radii 5 !" $0-12$ !" 0 centered on the quasar. Counts are summed in wedges of angular size $10^{\circ}$ and the contribution from the background is subtracted, as described in the text. The overdensity and its $1 \sigma$ uncertainty range are marked by the red line and the gray filled region, respectively. We highlight the position angle $-55^{\circ}$ and its complement $125^{\circ}$; this is where excess emission is detected, while Momjian et al. (2018) report jets at a position angle of $-61^{\circ}$. The ratio between this emission and its uncertainty is shown in the top panel; the signal is stronger than $3 \sigma$ at $-55^{\circ}$. Right: broadband sky image of PJ352-15, showing the extraction region (red, centered on $\theta=0^{\circ}$ ) and the 5!" $0-12 !^{\prime \prime} 0$ annulus. Photons detected outside this annulus are lightened for presentation purposes. The resolved radio structure has an overall linear extent of 0.28 (1.62 kpc), a size equivalent to one pixel in the X-ray image.

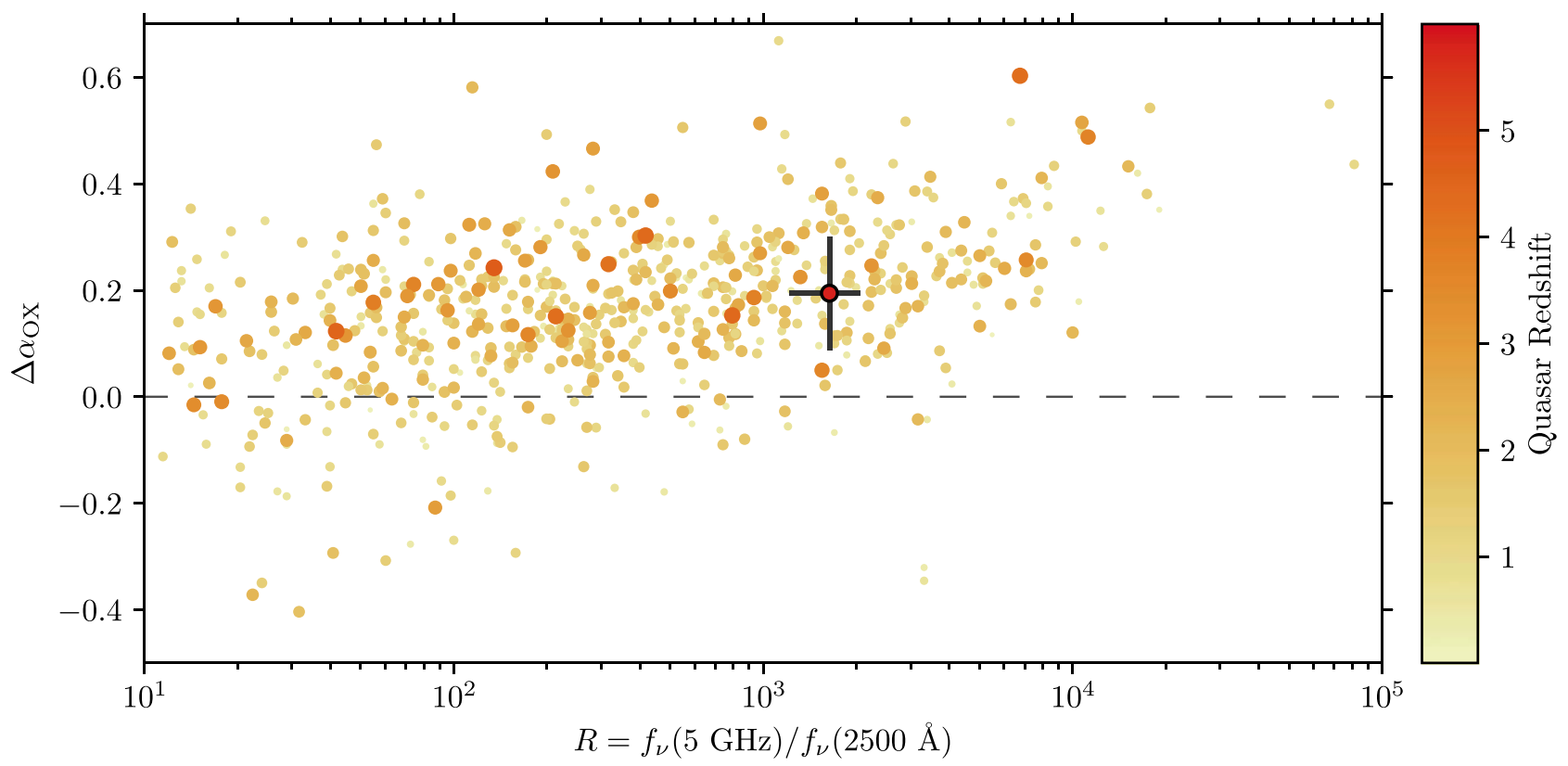

Figure 3. Offsets from the best-fit $\alpha_{\mathrm{OX}}$ relation as a function for radio-loudness for PJ352-15 and a sample of radio-loud quasars from Miller et al. (2011). Predicted values of $\alpha_{\mathrm{OX}}$ were calculated using the nominal relation of Lusso \& Risaliti (2016). Points are colored by redshift, with higher-redshift quasars being redder, as indicated on the color bar. Note that to conform to the catalog of Miller et al. (2011), radio-loudness in this figure is with respect to the monochromatic luminosity at $2500 \AA$, not $4400 \AA$.

offset from this prediction by $\Delta \alpha_{\mathrm{OX}}=0.19 \pm 0.11$. For this offset, the uncertainty accounts for changes in both the predicted and calculated $\alpha_{\mathrm{OX}}$ with changing $L_{\nu}(2500 \AA)$, but it does not include the uncertainties and intrinsic scatter in the best fit of Lusso \& Risaliti (2016). We show in Figure 3 how this value of $\Delta \alpha_{\text {OX }}$ compares to a broad sample of radio-loud quasars collected by Miller et al. (2011); note that, as the values of $R$ given in that work are based on $f_{\nu}(2500 \AA)$ and not 
$f_{\nu}(4400 \AA)$, we adjust the value of $R$ for PJ352-15 for this plot, again extrapolating from $M_{1450}$ assuming $\alpha_{\nu}=-0.5$.

As can be seen in Figure 3, PJ352-15 is not particularly $\mathrm{X}$-ray overluminous in the context of radio-loud quasars. For further comparison, we consider PSO J0309 +27, the $z=6.1$ blazar (Belladitta et al. 2020). That quasar has a similar UV luminosity to $\mathrm{PJ} 352-15\left(M_{1450}=-25.1\right)$ but is less X-ray overluminous $\left(\alpha_{\mathrm{OX}} \sim-1.6\right.$, converting from the $10 \mathrm{keV}$-based $\tilde{\alpha}_{\text {OX }}$ following Ighina et al. 2019). Miller et al. (2011) argued that the lack of redshift evolution seen in $\Delta \alpha_{\mathrm{OX}}$ for radio-loud quasars was indicative that IC/CMB only produced, at most, a minor contribution to the X-ray emission from these quasars. As it stands, neither of the two radio-loudest quasars known in the early universe show enough X-ray emission to contrast with that argument.

\subsection{Point Source or Extended Emission}

If jets are producing excess X-ray emission around PJ352 -15 , this could potentially manifest as a deviation from the predicted Chandra PSF, assuming the jets are sufficiently separated from the AGN. To test this possibility, we compare the observed quasar in three bands-soft $(0.5-2.0 \mathrm{keV})$, broad (0.5-7.0 keV), and hard (2.0-7.0 keV) - to a simulated PSF for these observations. Any significant deviations, as characterized by the binomial probability of structure in the observations being caused by random samplings of the PSF and background, would be indicative of X-ray emission outside of the central point source.

For all seven observations given in Table 1, we used MARX (Davis et al. 2012) to simulate our observed point source. The input source spectrum was derived from our best-fit parameters, but with a normalization 100 times larger to produce more photons. We did not include the effects of the readout streak or of pileup. Each observation was simulated 100 times, so that, in total, we produced $\sim 10,000$ simulated photons for every observed photon. We adopt an aspect blur of 0 !' 28 to account for uncertainties in the aspect solution, following the recommendations of the Chandra X-ray Center for ACIS-S observations. ${ }^{14}$ The simulations were facilitated with the CIAO task simulate_psf. Output events files were processed with the EDSER algorithm to be consistent with our observations. The observed quasar and simulated PSFs are shown in Figure 4; due to the use of EDSER in both data sets, bin sizes shown are half of an ACIS pixel, or 0." 246 on a side.

To evaluate the probability of deviations from the PSF, we compare our observations to these models, quantifying deviations using the binomial probability that the flux in a bin is consistent with the expectation of the overall background plus the contribution of the PSF at that location. We follow the methods outlined by Weisskopf et al. (2007) and Lansbury et al. (2014), namely, that the probability of $N$ counts arising by chance given an expectation of $N_{B}$ background counts can by expressed as

$$
P(N)=\sum_{i=N}^{\infty} \frac{N_{B}{ }^{i}}{i !} e^{-N_{B}} .
$$

Here, both $N_{B}$ and $N$ are evaluated in a $3 \times 3$ bin region centered on the bin of interest.

\footnotetext{
${ }^{14}$ https://cxc.cfa.harvard.edu/ciao/why/aspectblur.html
}

The results of this exercise are shown in the bottom panels of Figure 4. We detect no statistically significant deviation from the expectation of a quasar PSF with a stochastic background. While Chandra provides unparalleled angular resolution for $\mathrm{X}$-ray observations, our technique is nevertheless insensitive to features smaller than $\sim 4 \mathrm{kpc}$ at the redshift of PJ352-15 (0!'738). In comparison, the radio structures reported by Momjian et al. (2018) have a maximum angular extent of 0 ". 28.

\subsection{Potential Secondary Source}

In our initial analysis of the X-ray observations, we detected a secondary source near the position of the quasar. This source is located $\sim 19^{\prime \prime}(\sim 115 \mathrm{kpc}$ at the redshift of the quasar) from PJ352-15 at a position angle of $\sim 115^{\circ}$. This angle is roughly the counter-angle of the jets identified by Momjian et al. (2018) and the extended X-ray emission reported in Section 4.1. This source is dominated by hard energy photons, with a hardness ratio of $\mathcal{H R}=0.0 \pm 0.3$ from $\sim 20 \pm 5$ detected counts Chandra observations of PJ352-15 and this source are shown in the main panel of Figure 5. While there is no corresponding source in the Pan-STARRS1 imaging catalog (Chambers et al. $2016)$, this object is coincident with a faint source $(\sim 60 \mu \mathrm{Jy})$ in $3 \mathrm{GHz}$ imaging (Bañados et al. 2018b). From the deep X-ray and optical survey results of Marchesi et al. (2016), a source with this X-ray flux and hardness and with no optical flux in Pan-STARRS1 imaging being detected within 30" of PJ352 -15 is at least a $\sim 2 \sigma$ occurrence.

To constrain the properties of this source, we imaged the field in both the near-infrared and optical regimes. For the former, we used MOSFIRE (McLean et al. 2010, 2012) on the Keck I telescope to obtain $J$-band images. We observed the field on UT 2020 July 2 for 1452.4 s exposures (733.6 s total). Images were combined and processed with the AstrOmatic software suite (Bertin \& Arnouts 1996; Bertin et al. 2002; Bertin 2006) following standard reductions with IRAF. For optical imaging, we observed in the $r^{\prime}$ band with the GMOS-N imager (Hook et al. 2004) on the Gemini-North telescope as part of program GN-2020B-FT-101. Seven 423 s observations (2961 s total) in a filled-hexagon dither pattern were taken in queue mode on UT 2020 July 28. Data were reduced using the DRAGONS package (Labrie et al. 2019) following standard procedures. For both sets of observations, flux calibrations were performed by comparing other objects in the field to their reported photometry in 2MASS ( $J$; Skrutskie et al. 2006) and Pan-STARRS1 ( $r^{\prime}$; Chambers et al. 2016). Photometry was extracted in 1 ". 0 radius apertures, with backgrounds drawn in 3 ." $0-6$ ". 0 concentric annuli. Both observations are shown in the right of Figure 5.

At the redshift of PJ352-15, Ly $\alpha$ is at $\sim 8300 \AA$, and so all emission observed in the $r^{\prime}$ filter will be attenuated by absorption from intervening neutral gas (the Ly $\alpha$ forest). With the small projected separation between PJ352-15 and the secondary object, if they are both at $z \approx 5.83$ we would expect the same level of attenuation to be observed for both objects. As such, the color difference between the two objects, $\Delta_{r^{\prime}-J}$, should only reflect the innate color differences of the two objects. If no reasonable spectral model can explain the observed values of $\Delta_{r^{\prime}-J}$, then the objects must be being seen through different Ly $\alpha$ forests, and we can rule out this object as being at the redshift of PJ352-15.

From this photometry, we find that PJ352-15 has an $r^{\prime}-J$ color of $4.5 \mathrm{mag} \quad\left(r^{\prime}=25.8 \pm 0.2, \quad J=21.3 \pm 0.1\right.$; all 

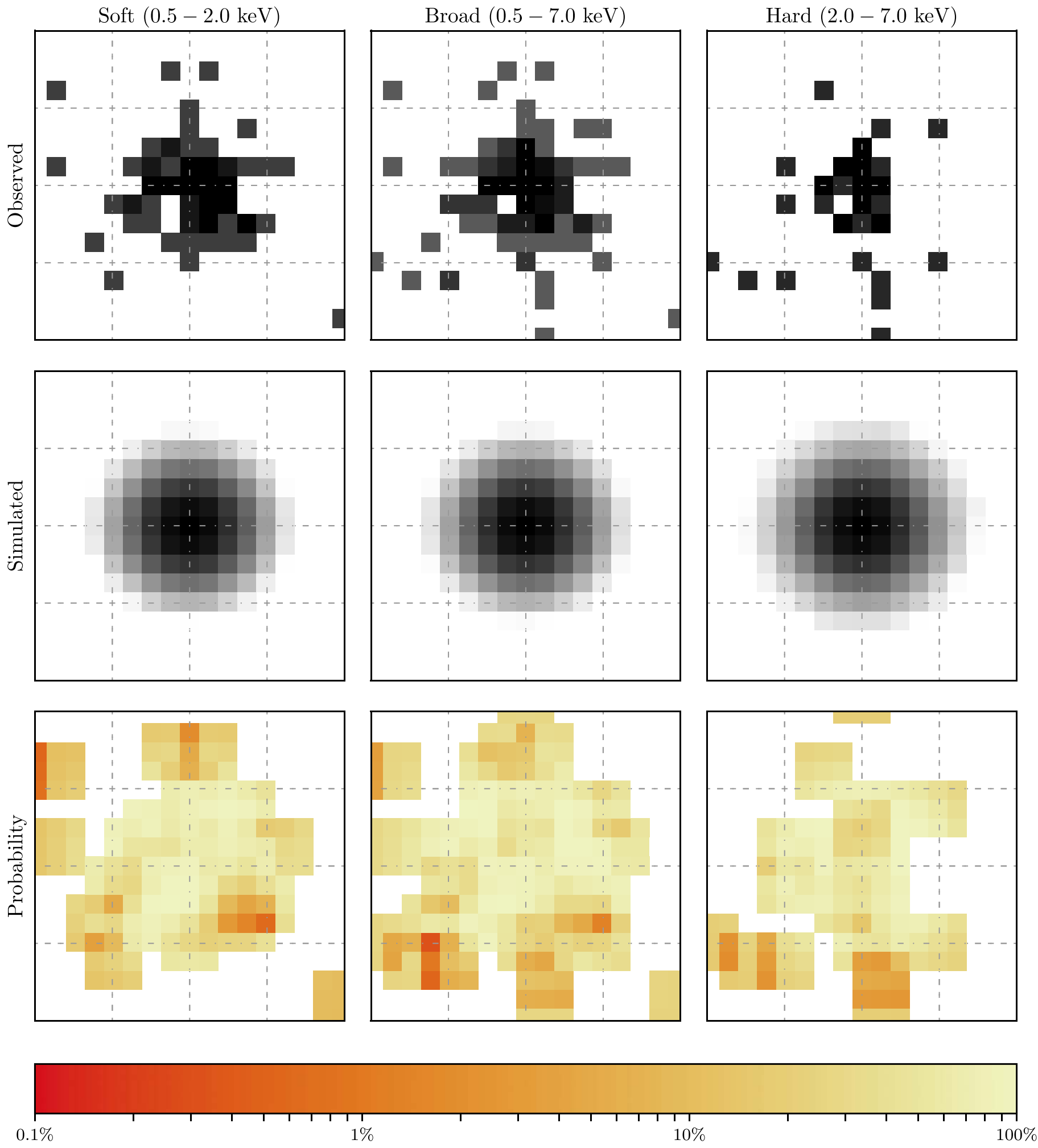

Binomial Probability of Signal Arising from PSF + Background

Figure 4. Comparisons between the observed quasar (top row) and a simulated PSF at that location (center row), with the binomial probability of any signal arising from the combined PSF and background (bottom row and color bar), as described in the text. The soft (left), broad (center), and hard (right) bands are independently evaluated. All images cover 4 " $0 \times 4$ " 0 fields of view centered on the peak of emission. No statistically significant deviation from the point source expectation is seen.

magnitudes are $\mathrm{AB})$. In contrast, the secondary object has a color of $r^{\prime}-J=0.3 \mathrm{mag}\left(r^{\prime}=24.6 \pm 0.2, J=24.3 \pm 0.2\right)$. As such, they cannot be at the same redshift and have the same spectral shape, so we rule out the possibility that this is a companion AGN. We also consider the possibility that this source is a hot spot on the counter-jet, and that we are seeing rest-frame optical and UV emission from star formation. Indeed, star formation triggered on radio jets has been seen for 

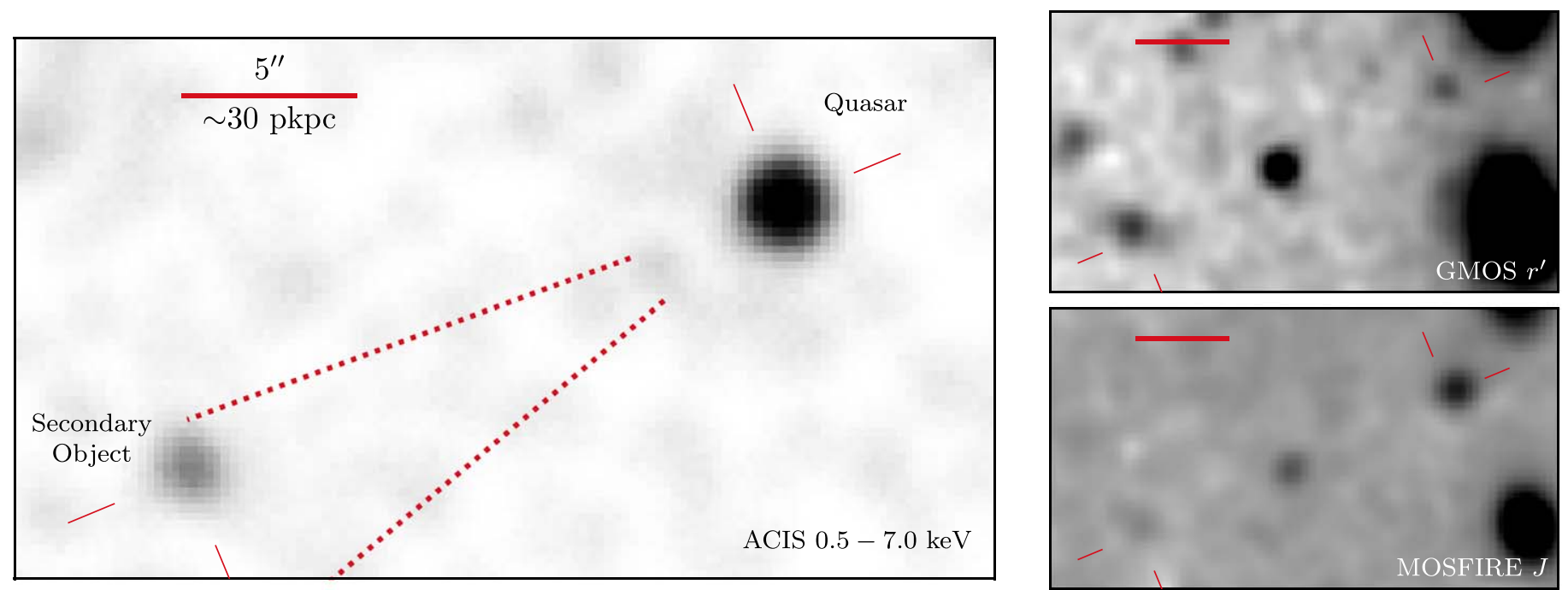

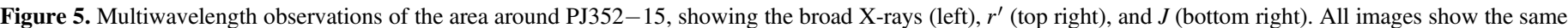

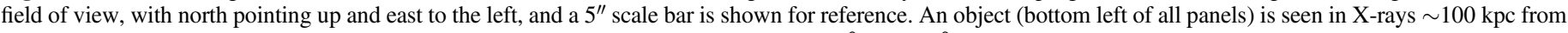

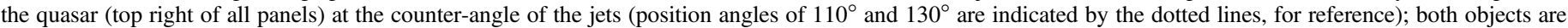

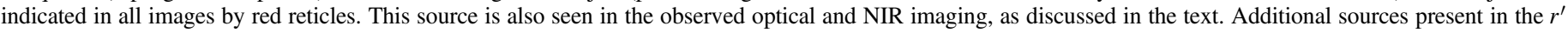
and $J$ images have no X-ray counterparts.

decades (van Breugel et al. 1985), and this effect is predicted to be common in the early universe (Croft et al. 2006), although previous results have not seen star formation triggered so far from the quasar (Nesvadba et al. 2020). To test this possibility, we use the synphot Python package to model the expected observed photometry of a quasar (using the template of Abazajian et al. 2004) and a 45,000 K blackbody (a rough approximation of a star-forming region), both at $z=5.83$. The expected color difference between a quasar and a star-forming region, $\Delta_{r^{\prime}-J} \sim 1.5$, is not consistent with what is observed $\left(\Delta_{r^{\prime}-J} \sim 4.2\right)$, which rules out the possibility that this object is at the redshift of the quasar.

\section{Properties of the Extended Emission}

In Section 4.1 we identified extended structure in the X-ray observations to the NW of the quasar position. While without a redshift measurement we cannot conclusively confirm a connection with PJ352-15, it is the strongest evidence of $\mathrm{X}$-ray emission from jets that we see around this quasar. Although only a limited number of photons with which to analyze this source are available, constraining the properties on this emission nevertheless sets limits on the strength of X-ray emission from one of the two radio-loudest quasars yet observed in the first billion years of the universe. To that end, we present the observed properties of the X-ray enhancement, assuming a redshift of $z=5.831$.

We extract a spectrum from a 2 ". 0 radius region centered on 23:29:36.35, $-15: 20: 09.6$. This region, which is $\sim 50 \mathrm{kpc}$ from the centroid of the quasar emission, is shown in Figure 6. We note that, while there is a galaxy to the NW of PJ352-15 visible in the optical imaging shown in Figure 5, the entirety of our extraction region is outside of the full extent of the galaxy in our $r^{\prime}$ and $J$ images, and no source is detected at this position in either band (to $3 \sigma$ limits of $J>24.3, r^{\prime}>26.2$ ). The extraction region has $7.8_{-3.5}^{+4.6}$ net counts, with a hardness ratio of $\mathcal{H} \mathcal{R}=0.1_{-0.4}^{+0.5}$. We compute a binomial probability of this emission being produced by the background for three bands: soft $(p=0.020)$, hard $(p=0.021)$, and broad $(p=0.0015)$.
Using PyXspec, we fit the observed emission to an absorbed power-law model, phabs $\times$ powerlaw. The best fit of this emission comes when $\Gamma \sim 4$ and $L_{2-10} \sim 3 \times$ $10^{44} \mathrm{erg} \mathrm{s}^{-1}$. With $\Gamma$ and the power-law normalization allowed to vary, we have two free parameters, and, with so few counts, the uncertainties on our fit are significant. Indeed, the hardness ratio, $\mathcal{H} \mathcal{R}=0.1_{-0.4}^{+0.5}$, is harder than that of PJ352-15 $\left(\mathcal{H} \mathcal{R}=-0.34_{-0.09}^{+0.08}\right)$, implying $\Gamma<2$. Despite the uncertainties, we do find some constraints on the observed emission. Assuming that $\Gamma \sim 2$ (e.g., Harris \& Krawczynski 2006), the $1 \sigma$ expectation of our fit is that this potential jet component has a luminosity of $L_{2-10} \lesssim 10^{44} \mathrm{erg} \mathrm{s}^{-1}$, and we can conclusively rule out jet emission of $L_{2-10} \gtrsim 10^{45} \mathrm{erg} \mathrm{s}^{-1}$. The full confidence intervals are shown in Figure 6.

As a comparison, we consider the sample of X-ray jet properties described by McKeough et al. (2016); this sample comes from Chandra imaging of 11 intermediate-redshift $(2.1 \lesssim z \lesssim 4.7)$ quasars known to host radio jets. In that work, McKeough et al. (2016) fit absorbed power laws to observed jet emission, including multiple regions for three quasars. From those fits we derive rest-frame luminosities for comparison using XSPEC. We note that, as the covariances between $\Gamma$ and normalization are not given for these fits, we calculated uncertainties in luminosity at the extreme values of both parameters, and therefore these uncertainties are most likely overestimates. The observed properties of these jets are shown in the right panel of Figure 6.

Assuming that this emission is not driven by a source with an extreme power-law spectral index $(\Gamma \gtrsim 5)$, PJ352-15 is not host to the most X-ray luminous jets in the $z>2$ universe. In contrast, for a steeper spectral slope, the high predicted luminosity is caused by extrapolating a poorly constrained spectrum into unobserved energy ranges (e.g., Connor et al. 2020) and is not indicative of an expected relation between $\Gamma$ and $L_{2-10}$. As can be seen in Figure 3, PJ352-15 is not an outlier in radio-loudness among the broad population of quasars; the only reason we would expect this emission to be large is due to the $(1+z)^{4}$ scaling of the CMB energy density. 

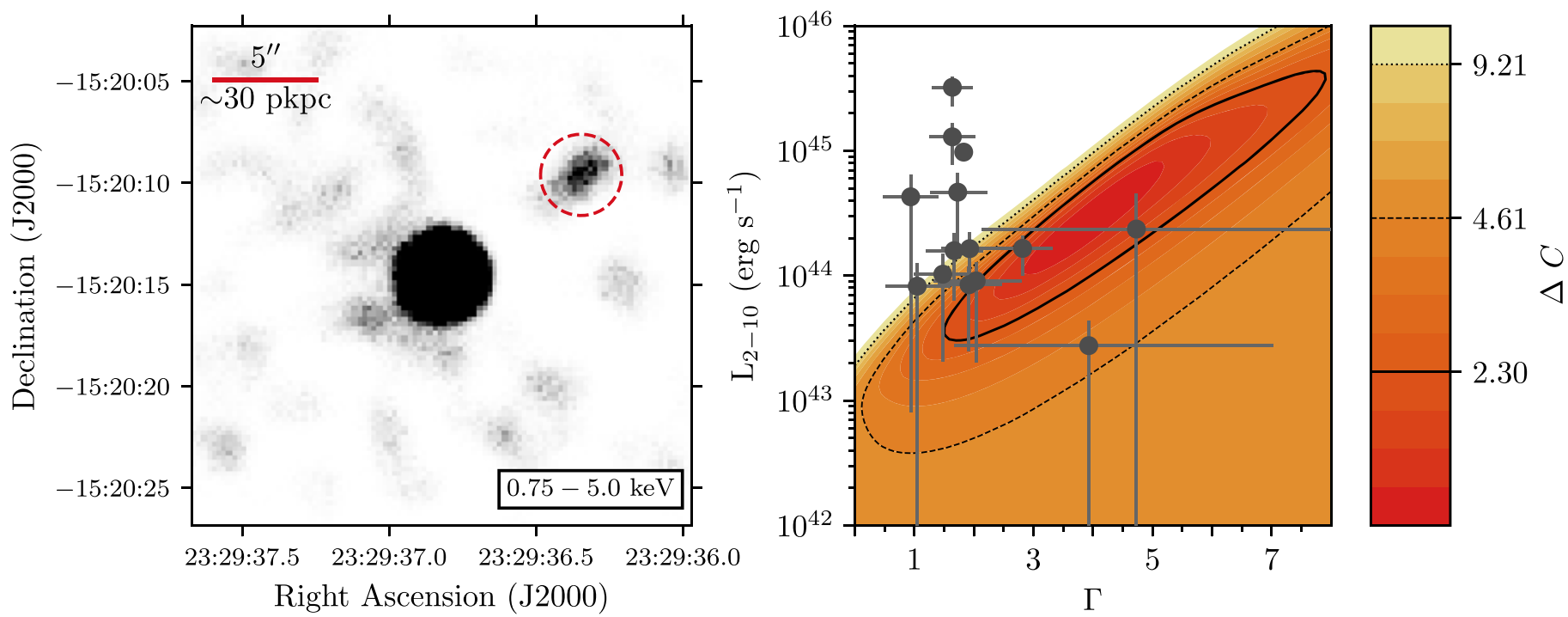

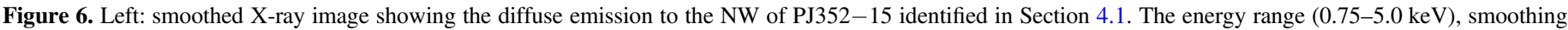

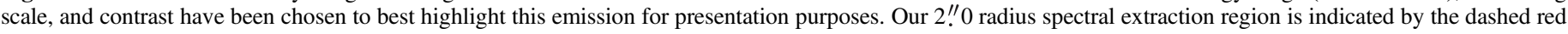

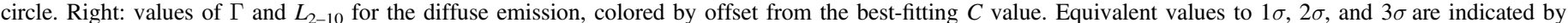

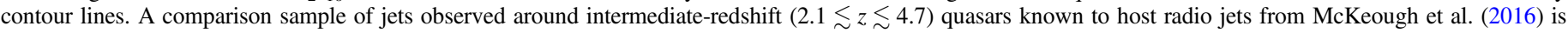
shown with gray points.

Following Lucchini et al. (2017), we calculate an equivalent magnetic field to the CMB's energy density as

$$
B_{\mathrm{CMB}}=3.26(1+z)^{2} \Gamma_{\text {jet }} \mu \mathrm{G},
$$

where $\Gamma_{\text {jet }}$ is the bulk Lorentz factor, finding $B_{\mathrm{CMB}}=$ $152 \Gamma_{\text {jet }} \mu \mathrm{G}$ at this redshift. If magnetic field strengths inferred from radio synchrotron measurements are less than this value then particle cooling should be dominated by X-ray IC processes. While values of $\Gamma_{\text {jet }} \sim 10$ have been seen at $z>5$ (e.g., Frey et al. 2015), the VLBI analysis of Momjian et al. (2018) did not find evidence for such fast outflows in this system, and so we adopt a conservative $\Gamma_{\text {jet }} \sim 1$. For a jet of relativistic particles with $\mathrm{X}$-ray emission caused by interaction with the CMB at redshift $z$ and radio emission driven by a magnetic field of strength $B$, the relative X-ray and radio flux densities can be found through

$$
\left(\frac{S_{r} \nu_{\mathrm{r}}}{S_{\mathrm{X}} \nu_{\mathrm{X}}}\right)=\frac{1}{2.89}\left(\frac{B}{\mu \mathrm{G}}\right)^{2}(1+z)^{-4},
$$

where $S_{\mathrm{X}}$ and $S_{\mathrm{r}}$ are the $\mathrm{X}$-ray and radio flux densities at observed frequencies $\nu_{\mathrm{X}}$ and $\nu_{\mathrm{r}}$, respectively, and $S_{\mathrm{r}} \propto \nu$ is assumed (Carilli \& Taylor 2002).

To constrain the radio emission, we examine archival observations of PJ352-15 taken with NSF's Karl G. Jansky Very Large Array (VLA) at $3 \mathrm{GHz}$ in 2018 January (Bañados et al. 2018a). We reprocessed these observations using standard techniques (e.g., Carilli et al. 2019). The resulting image, shown in Figure 7, has an rms noise of $9.5 \mu \mathrm{Jy}$ beam $^{-1}$ and a resolution of 2 !" $6 \times 1$ !! $4\left(\mathrm{PA}=-13^{\circ}\right)$. The secondary source to the SE of the quasar discussed in Section 4.4 has a nominal radio counterpart of $60 \mu \mathrm{Jy}$ beam $^{-1}$ ( $\sim 6 \sigma$ significance). At the location of the diffuse X-ray emission being discussed here, no radio emission is seen. If we assume this $\mathrm{X}$-ray emission is from IC upscattering of the CMB by relativistic electrons, and adopting flux densities of $<28.5 \mu \mathrm{Jy}(3 \sigma)$ at $3 \mathrm{GHz}$ and $58_{-43}^{+55} \times 10^{-12}$ Jy at $2.4 \times 10^{17} \mathrm{~Hz} \quad(1 \mathrm{keV} ;$ from the normalization of the X-ray spectral fit), then from Equation (6) we find an expected source magnetic field of $\lesssim 6 \mu \mathrm{G}$. This is a typical magnetic field strength for diffuse lobes of extragalactic radio sources (Miley 1980) and is significantly lower than the value of $\sim 3.5 \mathrm{mG}$ reported for the inner kiloparsec of the quasar by Momjian et al. (2018). Deeper radio observations are required to test if there is a radio counterpart to this possible X-ray knot around PJ352-15.

\section{Discussion}

In this work, we have presented analysis of X-ray observations of PJ352-15. In addition to the X-ray properties of the quasar itself, we also conducted a search for evidence of IC/CMB emission. Here, we discuss our results in the context of the broader perspective of high-redshift SMBH growth, AGN emission, and radio activity around quasars

One of the primary goals of this investigation was to search for evidence of extended jets as an indicator of enhanced accretion rates for high-redshift quasars. Jolley \& Kuncic (2008) presented a model (see also Ghisellini et al. 2013; Jolley et al. 2009) wherein a magnetic torque on the accretion disk extracts energy that is then injected into a magnetized jet. As the jet transports a minimal amount of mass but a more substantial amount of angular momentum, the net effect of this model is that the jet enhances the mass accretion rate, allowing for more significant growth than would be possible without the jet. Here, the model postulates that the jet is powered by extraction of energy and momentum from the disk (the Blandford-Payne processes; Blandford \& Payne 1982) rather than from the spin-energy of the black hole (the BlandfordZnajek mechanism; Blandford \& Znajek 1977), as is commonly invoked at lower redshifts. Because of this distinction, observed relations showing a decline in accretion rate with radio-loudness (e.g., Sikora et al. 2007) driven by conditions where jets are powered from the black hole spin (e.g., Sikora et al. 2013) are not pertinent to the ability of jets to enhance accretion rates in this model. 


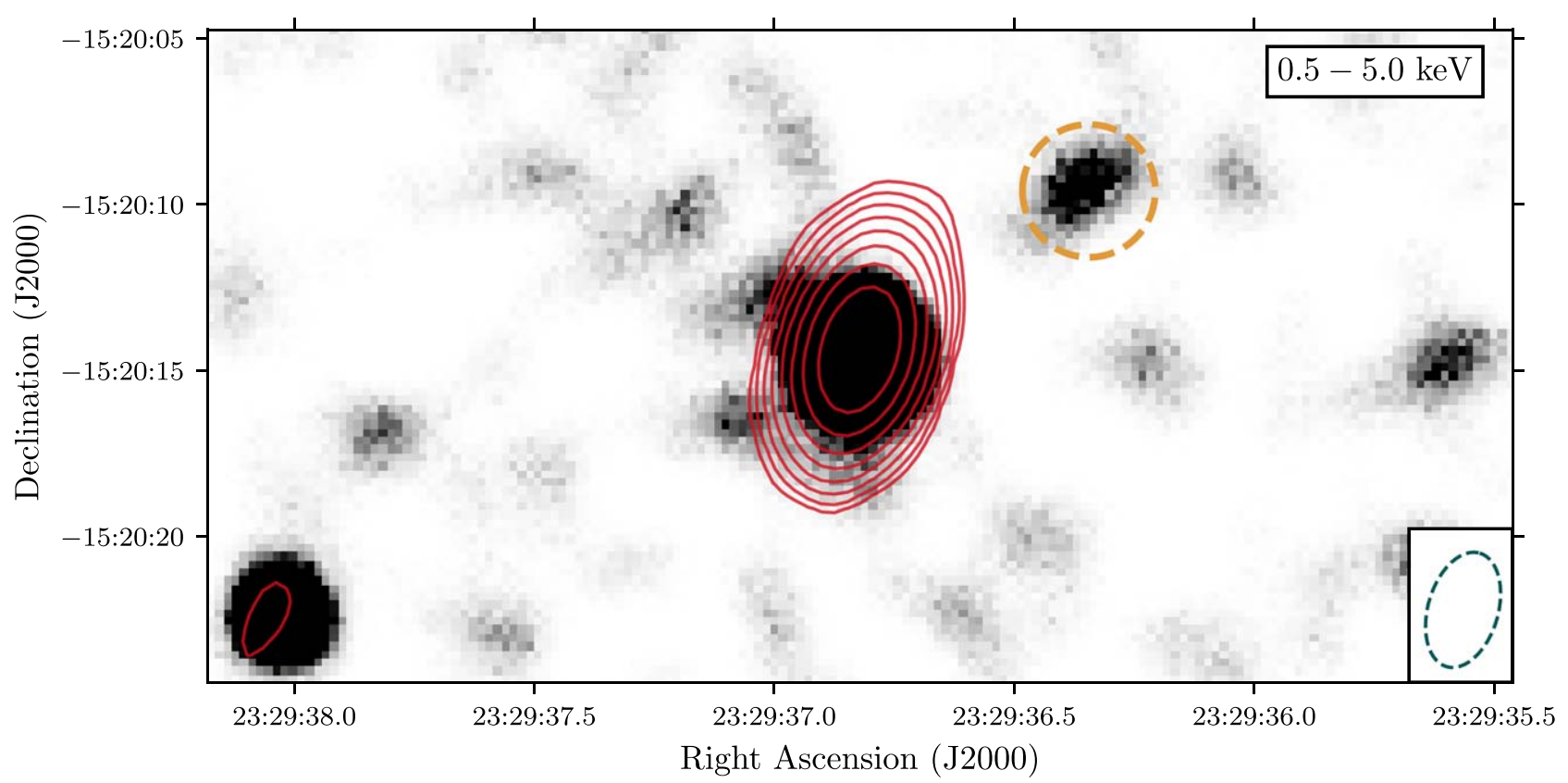

Figure 7. Smoothed X-ray emission with contours from $3 \mathrm{GHz}$ VLA observations overlaid. Contours are logarithmically spaced at levels of $f=2^{n} \times 9.5 \mu \mathrm{Jy}$ beam ${ }^{-1}$ for $n=1,2,3,4,5,6,7$, where $9.5 \mu \mathrm{Jy}$ beam ${ }^{-1}$ is the rms noise of these data. The secondary object discussed in Section 4.4 is shown in the bottom left of the figure, while the diffuse emission highlighted in Section 4.1 is indicated here by a dashed orange circle. Negative radio contours are not shown for clarity.

Potentially, this model of jet-enhanced accretion could complement the jet emission models of Garofalo et al. (2010), who present an evolutionary model for radio-loud AGN, starting with retrograde spins that produce BlandfordPayne jets and transitioning to Blandford-Znajek-dominated prograde systems. Prograde systems are more efficient at converting accreting mass to jet energy (Tchekhovskoy \& McKinney 2012), so the presence of large jets around a retrograde SMBH could indicate significant amounts of accretion have occurred. As black hole spin measurements at these redshifts are beyond the capabilities of current observatories (although see Gnedin et al. 2015, who postulate that ULAS J1120 +0641 at $z=7.08$ has a retrograde spin), a direct test of this hypothesis is not currently possible. Nevertheless, this scenario does present a case in which jet emission could enable the rapid buildup of SMBHs needed to reconcile observed quasar populations with theoretical models of seed formation.

Simulations provide some context to the role of jet feedback in regulating AGN growth in the early universe. Recently, Regan et al. (2019) simulated the formation of an SMBH through a metal-free supermassive star that directly collapses into a $\sim 10^{4} M_{\odot}$ black hole. Immediately after the collapse, mechanical feedback is turned on in the form of jets; these jets suppress accretion, driving the black hole growth to subEddington values, but their effect is limited to the inner $0.1 \mathrm{pc}$. In contrast, Takeo et al. (2020) simulated both radiative and mechanical feedback from a black hole of initial mass $10^{5} M_{\odot}$ and found that, after around one dynamical timescale $\left(\sim 8.4 \times 10^{6} \mathrm{yr}\right)$, a cascade of neutral gas along the equatorial region drives major outflows in the polar region. The balance found in this simulation is one of hyper-Eddington accretion and an evacuation of gas along the poles.

In contrast, the VLBI observations of jets presented by Momjian et al. (2018) provide direct evidence that the jets around PJ352-15 have, at least, extended beyond $1 \mathrm{kpc}$ from the SMBH itself. From the candidate jet emission reported here, the jets could have extended to $\sim 50 \mathrm{kpc}$, and the implied duration of jet launching is, assuming $v \sim 0.3 c$, of order $1 \mathrm{Myr}$. Recent interferometric observations of the $z=6.1$ blazar PSO J0309 +27 by Spingola et al. (2020) showed jets with projected sizes of hundreds of parsecs; while these structures are potentially larger given the viewing angle, they, in addition to the observations of Momjian et al. (2018), are in contrast to the limited effect of jets predicted by Regan et al. (2019) and further support the notion that these jets could be aiding in accretion.

Recently, Schwartz et al. (2020) reported two candidate $\mathrm{X}$-ray jets at intermediate redshifts $(z \sim 3.2)$ found using a technique similar to that employed here in Section 4.1. Although these jets are along a line connecting quasar cores to radio emission, they have no associated radio emission of their own. Similarly, X-ray emission associated with a $z \sim 4.3$ quasar reported by Siemiginowska et al. (2003) has no corresponding radio emission. For these three jets, the X-ray luminosities are of order $L_{2-10} \sim 10^{45} \mathrm{erg} \mathrm{s}^{-1}$, slightly higher than what we found here. While individually less compelling, the combination of all four reports of X-ray emission paints a picture wherein jets around intermediate- and high-redshift quasars may be hiding without associated detections of radio emission. If this is the case, only deep X-ray observations of radio-loud high-redshift quasars will enable a meaningful insight into this population.

\section{Summary}

We have presented deep ( $265 \mathrm{ks}$ ) X-ray observations of $\mathrm{PJ} 352-15$, one of the two radio-loudest quasars observed in the first billion years of the universe $(z>5.6$; see also Belladitta et al. 2020). As part of our analysis, we have also obtained optical and NIR imaging of the quasar and its surroundings. Our primary results are:

1. We fit the X-ray properties of the quasar itself, which is detected with over 100 counts; adopting an absorbed 
power-law model, we find the AGN emission is fit with $\Gamma=1.99_{-0.28}^{+0.29}$, and it has a corresponding unobscured luminosity of $L_{2-10}=1.26_{-0.33}^{+0.45} \times 10^{45} \mathrm{erg} \mathrm{s}^{-1}$.

2. In relation to the quasar's rest-frame UV emission, the observed X-ray luminosity is stronger than expected from the scaling relation for radio-quiet quasars of Lusso \& Risaliti (2016), with $\Delta \alpha_{\mathrm{OX}}=0.19 \pm 0.11$. However, in comparison to other radio-loud quasars, PJ352-15 is not an outlier in its X-ray loudness.

3. As radio jets have been observed around PJ352-15 (Momjian et al. 2018), we searched for evidence of these jets in X-rays. We found no excess X-ray emission in the core of the quasar, either in excess luminosity or in deviations from the expected PSF. We also investigated a nearby ( $\sim 19^{\prime \prime}$ offset) X-ray source along the predicted jet axis; follow-up optical and NIR imaging presented here rule out this emission being at the redshift of PJ352-15.

4. Through an analysis of the angular overdensity of emission, we identified a peak in the X-ray emission along the observed jet axis, corresponding to a $\gtrsim 3 \sigma$ deviation above the expectation. Further analysis of a 2 !" 0 radius region finds that this emission is unlikely to be associated with a background fluctuation, with a binomial probability of $P=0.0015$. As this emission is at the same position angle as the previously reported radio emission, we take this as tentative evidence for X-ray emission from the jets $\sim 50 \mathrm{kpc}$ from the position of the quasar.

5. Spectral analysis of the jet emission is limited by the small number of observed photons in the extraction region. However, assuming a spectral index similar to that found for other jet emission, the overall luminosity is $L_{2-10} \lesssim 10^{44} \mathrm{erg} \mathrm{s}^{-1}$.

6. The tentative detection of X-ray emission along the jet axis of PJ352-15 with no radio or optical counterpart is potentially indicative that this emission is being produced by IC interactions of jetted particles with the cosmic microwave background. Further, deeper observations are required to fully test this possibility.

The depth of the observations required to obtain these results -and the uncertainties still remaining-provide further support for the need for a new generation of X-ray observatories, namely, Athena (Nandra et al. 2013) and, hopefully, Lynx (Gaskin et al. 2019) and AXIS (Mushotzky et al. 2019).

The work of T.C. and D.S. was carried out at the Jet Propulsion Laboratory, California Institute of Technology, under a contract with NASA. T.C.'s research was supported by an appointment to the NASA Postdoctoral Program at the Jet Propulsion Laboratory, California Institute of Technology, administered by Universities Space Research Association under contract with NASA.

The scientific results reported in this article are based on observations made by the Chandra X-ray Observatory. This research has made use of software provided by the Chandra $\mathrm{X}$-ray Center $(\mathrm{CXC})$ in the application package CIAO. Based on observations obtained at the international Gemini Observatory (GN-2020B-FT-101), a program of NSFs NOIRLab, which is managed by the Association of Universities for Research in Astronomy (AURA) under a cooperative agreement with the National Science Foundation on behalf of the Gemini Observatory partnership: the National Science
Foundation (United States), National Research Council (Canada), Agencia Nacional de Investigación y Desarrollo (Chile), Ministerio de Ciencia, Tecnología e Innovación (Argentina), Ministério da Ciência, Tecnologia, Inovações e Comunicações (Brazil), and Korea Astronomy and Space Science Institute (Republic of Korea). Some of the data presented herein were obtained at the W. M. Keck Observatory, which is operated as a scientific partnership among the California Institute of Technology, the University of California, and the National Aeronautics and Space Administration. The Observatory was made possible by the generous financial support of the W. M. Keck Foundation.

This work was enabled by observations made from the Keck and Gemini-North telescopes, located within the Maunakea Science Reserve and adjacent to the summit of Maunakea. The authors wish to recognize and acknowledge the very significant cultural role and reverence that the summit of Maunakea has always had within the indigenous Hawaiian community. We are most fortunate to have the opportunity to conduct observations from this mountain and are grateful for the privilege of observing the universe from a place that is unique in both its astronomical quality and its cultural significance.

Facilities: CXO, Gemini:Gillett (GMOS-N), Keck:I (MOSFIRE).

Software: BEHR (Park et al. 2006), CIAO (Fruscione et al. 2006), MARX (Davis et al. 2012), PyFITS (Barrett \& Bridgman 1999), Synphot (STScI development Team 2018), WAVDETECT (Freeman et al. 2002), XSPEC (Arnaud 1996).

\section{ORCID iDs}

Thomas Connor (iD https://orcid.org/0000-0002-7898-7664 Eduardo Bañados (iD https://orcid.org/0000-0002-2931-7824

Daniel Stern (1D https://orcid.org/0000-0003-2686-9241

Chris Carilli (1) https://orcid.org/0000-0001-6647-3861

Andrew Fabian (1) https://orcid.org/0000-0002-9378-4072

Emmanuel Momjian (D) https://orcid.org/0000-0003-

3168-5922

Sofía Rojas-Ruiz (iD https://orcid.org/0000-0003-2349-9310

Roberto Decarli (i) https://orcid.org/0000-0002-2662-8803

Emanuele Paolo Farina (iD https://orcid.org/0000-0002-

6822-2254

Chiara Mazzucchelli (iD https://orcid.org/0000-0002-

5941-5214

Hannah P. Earnshaw (D) https://orcid.org/0000-00015857-5622

\section{References}

Abazajian, K., Adelman-McCarthy, J. K., Agüeros, M. A., et al. 2004, AJ, 128,502

Arnaud, K. A. 1996, adass V, 101, 17

Bañados, E., Carilli, C., Walter, F., et al. 2018a, ApJL, 861, L14

Bañados, E., Connor, T., Stern, D., et al. 2018b, ApJL, 856, L25

Bañados, E., Venemans, B. P., Decarli, R., et al. 2016, ApJS, 227, 11

Bañados, E., Venemans, B. P., Mazzucchelli, C., et al. 2018c, Natur, 553, 473

Bañados, E., Venemans, B. P., Morganson, E., et al. 2015, ApJ, 804, 118

Barrett, P. E., \& Bridgman, W. T. 1999, adass VIII, 172, 483

Belladitta, S., Moretti, A., Caccianiga, A., et al. 2020, A\&A, 635, L7

Bertin, E. 2006, adass XV, 351, 112

Bertin, E., \& Arnouts, S. 1996, A\&AS, 117, 393

Bertin, E., Mellier, Y., Radovich, M., et al. 2002, adass XI, 281, 228

Blandford, R. D., \& Payne, D. G. 1982, MNRAS, 199, 883

Blandford, R. D., \& Znajek, R. L. 1977, MNRAS, 179, 433

Breiding, P., Meyer, E. T., Georganopoulos, M., et al. 2017, ApJ, 849, 95

Brightman, M., Silverman, J. D., Mainieri, V., et al. 2013, MNRAS, 433, 2485 
Carilli, C. L., Perley, R. A., Dhawan, V., \& Perley, D. A. 2019, ApJL, 874, L32

Carilli, C. L., \& Taylor, G. B. 2002, ARA\&A, 40, 319

Cash, W. 1979, ApJ, 228, 939

Chambers, K. C., Magnier, E. A., Metcalfe, N., et al. 2016, arXiv:1612.05560

Chartas, G., Worrall, D. M., Birkinshaw, M., et al. 2000, ApJ, 542, 655

Connor, T., Bañados, E., Mazzucchelli, C., et al. 2020, ApJ, 900, 189

Connor, T., Bañados, E., Stern, D., et al. 2019, ApJ, 887, 171

Connor, T., Kelson, D. D., Mulchaey, J., et al. 2018, ApJ, 867, 25

Croft, S., van Breugel, W., de Vries, W., et al. 2006, ApJ, 647, 1040

Davis, J. E., Bautz, M. W., Dewey, D., et al. 2012, Proc. SPIE, 8443, 84431A

Fabian, A. C. 2016, AN, 337, 375

Fabian, A. C., Walker, S. A., Celotti, A., et al. 2014, MNRAS, 442, L81

Freeman, P. E., Kashyap, V., Rosner, R., \& Lamb, D. Q. 2002, ApJS, 138, 185

Frey, S., Paragi, Z., Fogasy, J. O., \& Gurvits, L. I. 2015, MNRAS, 446, 2921

Fruscione, A., McDowell, J. C., Allen, G. E., et al. 2006, Proc. SPIE, 6270, $62701 \mathrm{~V}$

Garmire, G. P., Bautz, M. W., Ford, P. G., Nousek, J. A., \& Ricker, G. R. J. 2003, Proc. SPIE, 4851, 28

Garofalo, D., Evans, D. A., \& Sambruna, R. M. 2010, MNRAS, 406, 975

Gaskin, J. A., Swartz, D. A., Vikhlinin, A., et al. 2019, JATIS, 5, 021001

Gehrels, N. 1986, ApJ, 303, 336

Ghisellini, G., Haardt, F., Ciardi, B., et al. 2015, MNRAS, 452, 3457

Ghisellini, G., Haardt, F., Della Ceca, R., Volonteri, M., \& Sbarrato, T. 2013, MNRAS, 432, 2818

Gilli, R., Mignoli, M., Peca, A., et al. 2019, A\&A, 632, A26

Gnedin, Y. N., Mikhailov, A. G., \& Piotrovich, M. Y. 2015, AN, 336, 312

Harris, D. E., \& Krawczynski, H. 2006, ARA\&A, 44, 463

HI4PI Collaboration, Ben Bekhti, N., Flöer, L., et al. 2016, A\&A, 594, A116

Hook, I. M., Jørgensen, I., Allington-Smith, J. R., et al. 2004, PASP, 116, 425

Ighina, L., Caccianiga, A., Moretti, A., et al. 2019, MNRAS, 489, 2732

Inayoshi, K., Visbal, E., \& Haiman, Z. 2020, ARA\&A, 58, 27

Jiang, L., McGreer, I. D., Fan, X., et al. 2016, ApJ, 833, 222

Jimenez-Gallardo, A., Massaro, F., Prieto, M. A., et al. 2020, ApJS, 250, 7

Jolley, E. J. D., \& Kuncic, Z. 2008, MNRAS, 386, 989

Jolley, E. J. D., Kuncic, Z., Bicknell, G. V., \& Wagner, S. 2009, MNRAS, 400, 1521

Kellermann, K. I., Sramek, R., Schmidt, M., Shaffer, D. B., \& Green, R. 1989 AJ, 98, 1195

Labrie, K., Anderson, K., Cárdenes, R., Simpson, C., \& Turner, J. E. H. 2019, ASPC, 523, 321

Lansbury, G. B., Alexander, D. M., Del Moro, A., et al. 2014, ApJ, 785, 17

Li, J., Kastner, J. H., Prigozhin, G. Y., et al. 2004, ApJ, 610, 1204

Li, J., Kastner, J. H., Prigozhin, G. Y., \& Schulz, N. S. 2003, ApJ, 590, 586

Lucchini, M., Tavecchio, F., \& Ghisellini, G. 2017, MNRAS, 466, 4299

Lusso, E., \& Risaliti, G. 2016, ApJ, 819, 154

Marchesi, S., Civano, F., Elvis, M., et al. 2016, ApJ, 817, 34

Marshall, H. L., Gelbord, J. M., Worrall, D. M., et al. 2018, ApJ, 856, 66

Matsuoka, Y., Iwasawa, K., Onoue, M., et al. 2019, ApJ, 883, 183

Mazzucchelli, C., Bañados, E., Venemans, B. P., et al. 2017, ApJ, 849, 91

McKeough, K., Siemiginowska, A., Cheung, C. C., et al. 2016, ApJ, 833, 123

McLean, I. S., Steidel, C. C., Epps, H., et al. 2010, Proc. SPIE, 7735, 77351E

McLean, I. S., Steidel, C. C., Epps, H. W., et al. 2012, Proc. SPIE, 8446, $84460 \mathrm{~J}$
Medvedev, P., Sazonov, S., Gilfanov, M., et al. 2020, MNRAS, 497, 1842

Meyer, E. T., Georganopoulos, M., Sparks, W. B., et al. 2015, ApJ, 805, 154 Miley, G. 1980, ARA\&A, 18, 165

Miller, B. P., Brandt, W. N., Schneider, D. P., et al. 2011, ApJ, 726, 20

Momjian, E., Carilli, C. L., Bañados, E., Walter, F., \& Venemans, B. P. 2018, ApJ, 861, 86

Mortlock, D. J., Warren, S. J., Venemans, B. P., et al. 2011, Natur, 474, 616

Mushotzky, R., Aird, J., Barger, A. J., et al. 2019, BAAS, 51, 107

Nandra, K., Barret, D., Barcons, X., et al. 2013, arXiv:1306.2307

Nanni, R., Gilli, R., Vignali, C., et al. 2018, A\&A, 614, A121

Napier, K., Foord, A., Gallo, E., et al. 2020, MNRAS, 498, 1550

Nesvadba, N. P. H., Bicknell, G. V., Mukherjee, D., \& Wagner, A. Y. 2020, A\&A, 639, L13

Paliya, V. S., Ajello, M., Cao, H. M., et al. 2020, ApJ, 897, 177

Park, T., Kashyap, V. L., Siemiginowska, A., et al. 2006, ApJ, 652, 610

Pons, E., McMahon, R. G., Simcoe, R. A., et al. 2019, MNRAS, 484, 5142

Reddy, K., Georganopoulos, M., \& Meyer, E. T. 2021, ApJS, 253, 37

Reed, S. L., McMahon, R. G., Martini, P., et al. 2017, MNRAS, 468, 4702

Regan, J. A., Downes, T. P., Volonteri, M., et al. 2019, MNRAS, 486, 3892

Schwartz, D. A., Marshall, H. L., Lovell, J. E. J., et al. 2000, ApJL, 540, 69

Schwartz, D. A., Siemiginowska, A., Snios, B., et al. 2020, ApJ, 904, 57

Siemiginowska, A., Smith, R. K., Aldcroft, T. L., et al. 2003, ApJL, 598, L15

Siemiginowska, A., Stawarz, Ł., Cheung, C. C., et al. 2007, ApJ, 657, 145

Sikora, M., Stasińska, G., Kozieł-Wierzbowska, D., Madejski, G. M., \& Asari, N. V. 2013, ApJ, 765, 62

Sikora, M., Stawarz, Ł., \& Lasota, J.-P. 2007, ApJ, 658, 815

Simionescu, A., Stawarz, Ł., Ichinohe, Y., et al. 2016, ApJL, 816, L15

Skrutskie, M. F., Cutri, R. M., Stiening, R., et al. 2006, AJ, 131, 1163

Spingola, C., Dallacasa, D., Belladitta, S., et al. 2020, A\&A, 643, L12

Steffen, A. T., Strateva, I., Brandt, W. N., et al. 2006, AJ, 131, 2826

Stern, D., Djorgovski, S. G., Perley, R. A., de Carvalho, R. R., \& Wall, J. V. 2000, AJ, 119, 1526

Strateva, I. V., Brandt, W. N., Schneider, D. P., Vanden Berk, D. G., \& Vignali, C. 2005, AJ, 130, 387

STScI development Team 2018, synphot: Synthetic Photometry using Astropy, Astrophysics Source Code Library, ascl:1811.001

Takeo, E., Inayoshi, K., \& Mineshige, S. 2020, MNRAS, 497, 302

Tchekhovskoy, A., \& McKinney, J. C. 2012, MNRAS, 423, L55

van Breugel, W., Filippenko, A. V., Heckman, T., \& Miley, G. 1985, ApJ, 293, 83

Vignali, C., Brandt, W. N., \& Schneider, D. P. 2003, AJ, 125, 433

Vito, F., Brandt, W. N., Bauer, F. E., et al. 2019a, A\&A, 628, L6

Vito, F., Brandt, W. N., Bauer, F. E., et al. 2019b, A\&A, 630, A118

Wachter, K., Leach, R., \& Kellogg, E. 1979, ApJ, 230, 274

Wang, F., Fan, X., Yang, J., et al. 2021a, ApJ, 908, 53

Wang, F., Yang, J., Fan, X., et al. 2019, ApJ, 884, 30

Wang, F., Yang, J., Fan, X., et al. 2021b, ApJL, 907, L1

Weisskopf, M. C., Wu, K., Trimble, V., et al. 2007, ApJ, 657, 1026

Worrall, D. M., Birkinshaw, M., Marshall, H. L., et al. 2020, MNRAS, 497, 988

Wu, X.-B., Wang, F., Fan, X., et al. 2015, Natur, 518, 512

Yang, J., Wang, F., Fan, X., et al. 2019, AJ, 157, 236

Yang, J., Wang, F., Fan, X., et al. 2020, ApJL, 897, L14 